\title{
A Parametric Geometry Computational Fluid Dynamics (CFD) Study Utilizing Design of Experiments (DOE)
}

\author{
Ray D. Rhew ${ }^{*}$ and Peter A. Parker! \\ NASA Langley Research Center, Hampton, Virginia 23681
}

\begin{abstract}
Design of Experiments (DOE) techniques were applied to the Launch Abort System (LAS) of the NASA Crew Exploration Vehicle (CEV) parametric geometry Computational Fluid Dynamics (CFD) study to efficiently identify and rank the primary contributors to the integrated drag over the vehicles ascent trajectory. Typical approaches to these types of activities involve developing all possible combinations of geometries changing one variable at a time, analyzing them with CFD, and predicting the main effects on an aerodynamic parameter, which in this application is integrated drag. The original plan for the LAS study team was to generate and analyze more than 1000 geometry configurations to study 7 geometric parameters. By utilizing DOE techniques the number of geometries was strategically reduced to 84 . In addition, critical information on interaction effects among the geometric factors were identified that would not have been possible with the traditional technique. Therefore, the study was performed in less time and provided more information on the geometric main effects and interactions impacting drag generated by the LAS. This paper discusses the methods utilized to develop the experimental design, execution, and data analysis.

* Research Engineer, Aeronautics Systems Engineering Branch, Mail Stop 435

! Research Scientist, Aeronautics Systems Engineering Branch, Mail Stop 238
\end{abstract}

\section{Introduction}

The Explorations Systems Mission Directorate (ESMD) is tasked with designing and developing the system of vehicles to fulfill the new space architecture, see reference 1 for more details on the background of the tasks that are summarized here. The first vehicle in the architecture is the Ares I Crew Launch Vehicle (CLV), which will be used to launch astronauts to low earth orbit. One organizational element of the Ares I CLV is the Aerodynamics Panel that is responsible for assuring that the aerodynamic design satisfies the Ares I CLV requirements. To meet these requirements the approach being taken includes a combination of wind tunnel experiments and CFD analysis. One of the objectives of the CFD analysis is to provide a rapid assessment of possible outer mold line (OML) design changes. This study, a parametric geometry CFD study utilizing DOE, fulfills that objective.

The Ares I CLV configuration, designated design and analysis cycle zero (DAC-0), was established in January 2006. A picture of a $0.548 \%$-scale wind tunnel model of this configuration is shown in figure 1 with three primary elements labeled, the Launch Abort System (LAS), Crew Explorations Vehicle (CEV), and the upper stage. Preliminary wind tunnel testing of this configuration revealed potential aerodynamic improvement during the ascent phase of the LAS. Therefore, a study was undertaken to understand this 
potential improvement using CFD and wind tunnel testing. The first phase of the study was the CFD study that is discussed in this paper.

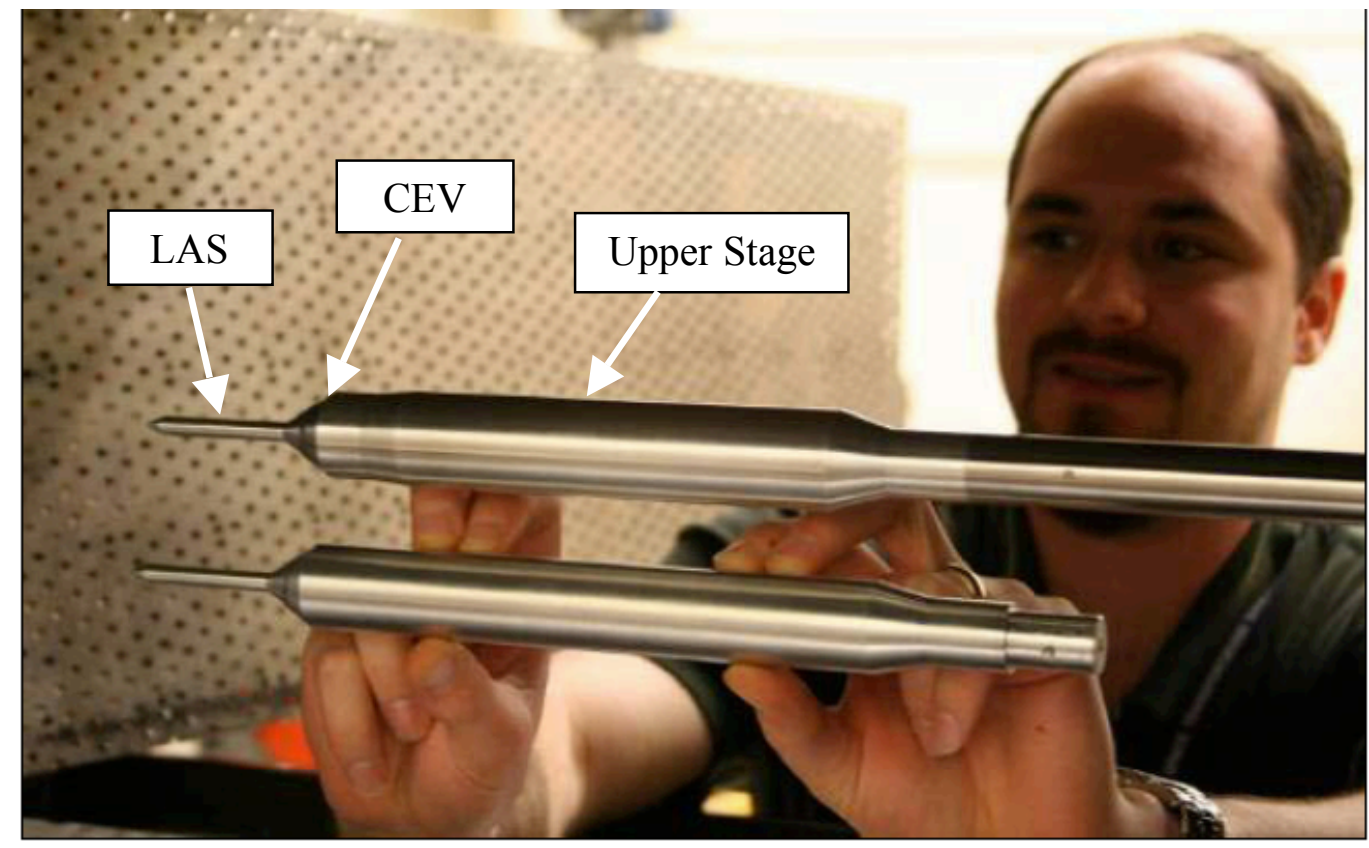

Figure 1. 0.548\%-scale DAC-0 Wind Tunnel Model

The design space of the geometry trade study is large due to the number of geometric parameters. A decision was made by the project to utilize Design of Experiments (DOE) methodologies to perform the study in the most effective manner. A classical DOE methodology emphasizes efficiently designing experiments to attain as much information as possible with each run (experimental unit). This is achieved by simultaneously changing multiple factors between experimental runs as opposed to changing one-factorat-a-time (OFAT). By employing a DOE methodology additional information can be obtained from the experimental runs, such as factor interactions that may be overlooked during traditional OFAT experiments. For this study it was determined that the traditional OFAT approach would have required approximately 1,566 configurations to be generated to obtain the information required for assessment of the aerodynamic drag effects of the LAS on the Ares I CLV DAC-0 configuration. However, only 84 configurations were needed using the DOE approach to answer the questions and objectives of the study. In addition, information on interactions was obtained that would not have been available in the OFAT approach. Reference 2 provides detail on DOE and its benefits as applied to product and process development and optimization.

\section{Experimental Design Development}

Experimental design planning is vital to utilize all of the pre-experimental information available. An excellent discussion on experimental design planning is provided in reference 3. For the LAS study, meetings and discussions were held with subject matter experts (SME) in experimental aerodynamics, CFD, and members of the LAS aerodynamics team to complete the design sheet and enable efficient development of the 
experimental design. Below are the contents of the guide sheet for this activity. At the beginning of each section in italics is a description of the information needed followed by the results of the SME meetings. Note that blank columns have been retained to highlight additional information that may be available in other experimental cases.

1) Objectives of the Experiment: Define the objectives such that they are unbiased, specific, measurable, and of practical consequence. Determine the nature of the experiment, either exploratory, verification of requirements, or an optimization. Also include the risks (consequences) of making an inferential mistake from the data.

Using CFD, identify the important (and unimportant) LAS parameters (factors) that influence the integrated drag (response). Quantify the relative magnitude of the factor effects and rank-order them in terms of their contribution to the integrated drag. The practical consequences of this experiment are: (1) to strategically guide future wind tunnel experiments in terms of identifying specific configurations of interest, to specify the number of configurations and associated fabrication of wind tunnel model components, and to specify appropriate experimental data volume, (2) to guide additional CFD studies, possibly requiring a higher-fidelity model of the factorresponse relationship, and (3) to become a smart-buyer in evaluating external proposals for CEV/CLV. We classify this first experiment as exploratory in nature, and may lead to optimization. Risks of a poorly designed experiment include inefficient use of CFD resources, too many or not enough wind tunnel experiments to answer the research questions, and ultimately poor drag performance of the vehicle in flight.

2) Relevant Background: Gather information that is relevant to the objectives that will help guide the experimental design such as previous test entries, and computational results prior to the experiment.

Recent wind tunnel testing has revealed that two configurations of the LAS design have considerable impact on the integrated drag of the CEV. Therefore, this study has been undertaken to assess how the design variables influence the integrated drag.

3) Response Variables, Measures of Performance: Identify response variables, variables that are indicators of the performance of the system under investigation, and the methods of measuring them. In this activity examples of potential parameters are aerodynamic coefficients and derivates of coefficients. Table 1 lists the response variables and associated information (if known) such as units, ranges, precision (and the source of this information), priority, type of parameter (continuous or discrete) and how the parameter will be obtained. 


\begin{tabular}{|c|l|c|c|c|c|c|c|}
\hline $\begin{array}{c}\text { Variable } \\
\text { (abbrev.) }\end{array}$ & Units & $\begin{array}{c}\text { Range } \\
\text { Low }\end{array}$ & $\begin{array}{c}\text { Range } \\
\text { High }\end{array}$ & $\begin{array}{c}\text { Precision } \\
\text { (source) }\end{array}$ & $\begin{array}{c}\text { Priority } \\
\mathbf{( 1 ~ h i g h )}\end{array}$ & $\begin{array}{c}\text { Type } \\
\mathbf{( c ,} \mathbf{d})\end{array}$ & Source \\
\hline $\begin{array}{c}\text { Int_Drag } \\
\text { (Integrated } \\
\text { Drag) }\end{array}$ & & & & $\begin{array}{c}\text { computational, } \\
\text { deterministic }\end{array}$ & 1 & continuous & $\begin{array}{c}\text { CFD } \\
\text { combined } \\
\text { with } \\
\text { trajectory }\end{array}$ \\
\hline
\end{tabular}

\section{Table 1. Response Variable}

4) Factors, Control Variables: These factors are measurable, controllable, and thought to be influential. Their effects are of primary interest in the current experiment. In this activity examples of potential parameters are: alpha, beta, Mach, Reynolds Number, model configurations.

Table 2 lists the factors and associated information (if known) such as units, ranges, type of parameter (continuous or categorical) and restrictions on setting the parameter. Figure 2 defines the factors on the LAS drawing. As discussed earlier each configuration will be evaluated over 10 pre-defined Mach numbers: 0.7, 0.9, 0.95, $1.05,1.1,1.3,1.46,1.96,2.74$, and 4.0. An integrated drag coefficient was developed as a weighted sum of coefficients from different Mach numbers based on dynamic pressure and time in order to calculate a single drag value for each experimental run.

\begin{tabular}{|c|c|c|c|c|c|c|}
\hline Label & $\begin{array}{c}\text { Factor } \\
\text { (abbrev.) }\end{array}$ & Units & $\begin{array}{l}\text { Range } \\
\text { Low }\end{array}$ & $\begin{array}{l}\text { Range } \\
\text { High }\end{array}$ & Type & Restrictions \\
\hline A & $\begin{array}{c}\text { TowerLen } \\
\text { (Tower Length) }\end{array}$ & inches & 326 & 490 & Continuous & \\
\hline B & $\begin{array}{c}\text { TowerDia } \\
\text { (Tower Diameter) }\end{array}$ & inches & 26 & 46 & Continuous & \\
\hline $\mathrm{C}$ & $\begin{array}{c}\text { TipFineRatio } \\
\text { (Tip Finess Ratio) }\end{array}$ & $1 / \mathrm{d}$ ratio & 0.5 & 2 & Continuous & \\
\hline D & $\begin{array}{l}\text { FlareDiaRatio } \\
\text { (Flare Diameter } \\
\text { Ratio) }\end{array}$ & $\begin{array}{c}\% \text { of } \\
\text { TowDia }\end{array}$ & 1.5 & 2.5 & Continuous & \\
\hline $\mathrm{E}$ & FlareAngle & deg & 25 & 45 & Continuous & \\
\hline $\mathrm{F}$ & $\begin{array}{l}\text { FlareLoc (Flare } \\
\text { Location) }\end{array}$ & ht/TowLen & 0.4 & 0.8 & Continuous & \\
\hline G & TipShape & & ellipse & sphere/cone & categorical & 2 levels \\
\hline
\end{tabular}

Table 2. Factors/Control Variables 

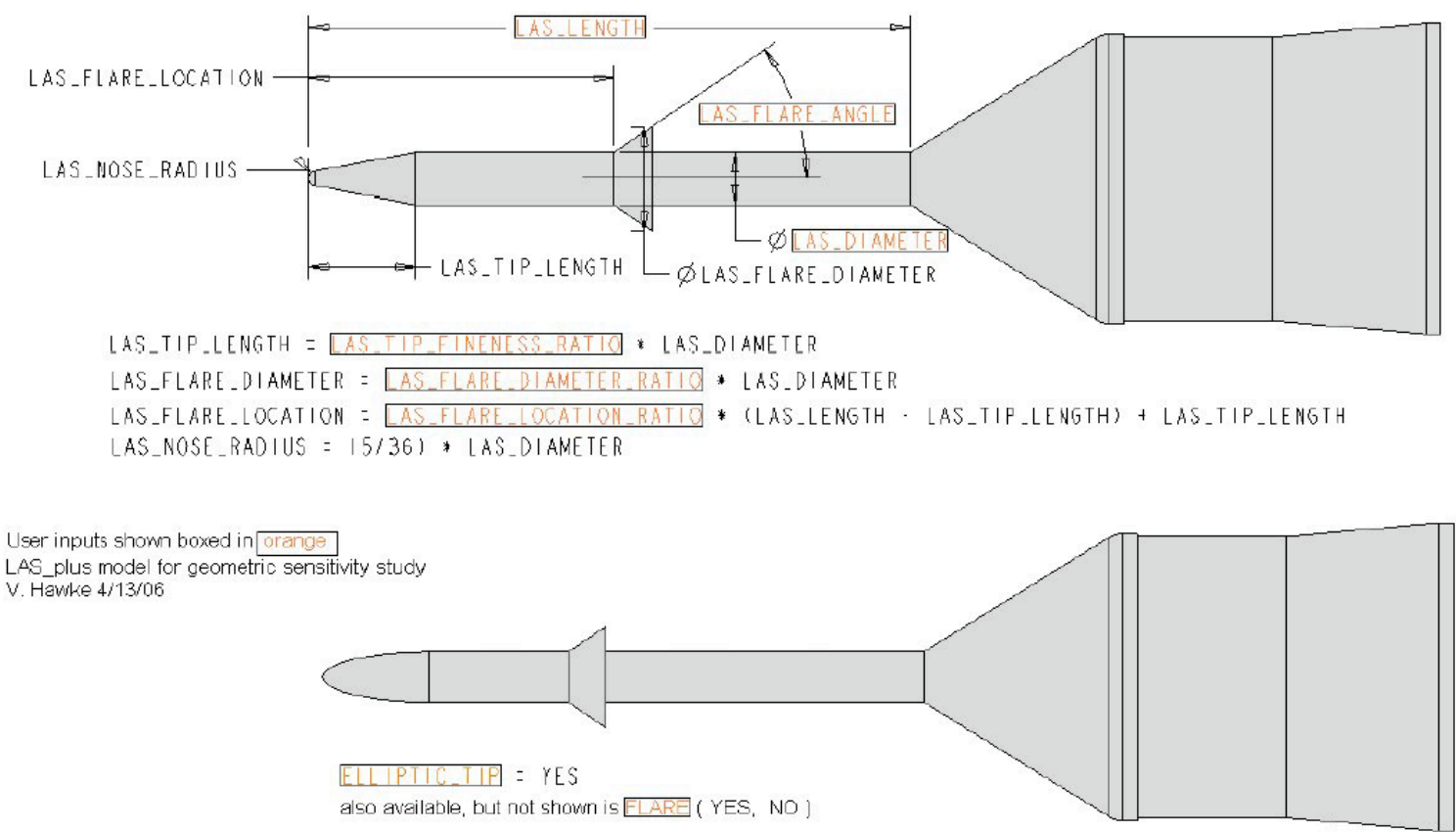

Figure 2. LAS with Factor Labels

5) Factors to be "held constant": These are Additional factors that are controllable, and whose effects are not of interest in this experiment. Potential factors are additional model configuration variables, and test facilities. Table 3 lists these factors.

\begin{tabular}{|c|l|l|l|l|}
\hline $\begin{array}{c}\text { Factor } \\
\text { (abbrev.) }\end{array}$ & Units & $\begin{array}{c}\text { Range } \\
\text { Low }\end{array}$ & $\begin{array}{c}\text { Range } \\
\text { High }\end{array}$ & Comments \\
\hline Re & & & $\begin{array}{l}\text { Flight Reynolds number will be used in this } \\
\text { investigation. Two other levels reflecting wind } \\
\text { tunnel testing may be considered as a follow-up. }\end{array}$ \\
\hline CFD code & & & A single CFD code will be used by the effort. \\
\hline $\begin{array}{c}\text { Axes-Sym } \\
\text { Geometry }\end{array}$ & & & & $\begin{array}{l}\text { The CFD model will be axes-sym (angle-of- } \\
\text { attack }=0 \text { degrees) }\end{array}$ \\
\hline
\end{tabular}

Table 3. Factors to be held constant

6) Nuisance factors: These factors are not controlled and are not of primary interest. They cannot be deliberately set to a constant level. For example: time varying effects, test article contamination, wear, operator (shift) bias, temperature.

For this activity the SMEs provide only one possible nuisance factor as listed in table 4. 


\begin{tabular}{|c|l|l|l|l|}
\hline $\begin{array}{c}\text { Factor } \\
\text { (abbrev.) }\end{array}$ & Units & $\begin{array}{c}\text { Range } \\
\text { Low }\end{array}$ & $\begin{array}{c}\text { Range } \\
\text { High }\end{array}$ & Comments \\
\hline $\begin{array}{c}\text { CFD solution } \\
\text { error }\end{array}$ & & & & $\begin{array}{l}\text { Numerical error in the CFD solutions has been } \\
\text { considered negligible and will not be estimated. } \\
\text { No replicates will be performed. }\end{array}$ \\
\hline
\end{tabular}

Table 4. Nuisance Factors

7) Interactions: Any prior knowledge of the effect of one factor being dependent on the level of another is important to ensuring it is captured in the design.

In this activity prior knowledge of the system indicates that two-way interactions and possibly three-way interactions are important to estimate. Specific interactions have not been identified.

8) Restrictions: This information is very important in developing an efficient design. Examples of restrictions are: time, number of experimental units, hard-to-change (HTC) factors, unsafe conditions that limit the factor levels, blocking and randomization, factor level settings, number of levels, or specific levels.

A parametric model is being generated, which makes all factors equally easy to change. Once the geometry is defined, the CFD can be performed rather quickly. So, minimizing the number of configuration factors (HTC) that define the geometry is desirable. In contrast, setting multiple Mach number levels for a given configuration is easier to manipulate. Due to the deterministic nature of the experiment, blocking and randomization of run ordering is unnecessary.

9) Design Preferences: Determine if there are any particular preferences on the statistical design that will aide in the presentation of results or potential findings.

Based on the objectives it was decided that two-level designs with a center point are desirable. The design needs to support the estimation of two-way interactions (2FI), with a desire to consider three-way (3FI) interactions as well.

10) Analysis and Presentation techniques preferred: This is very important to ensure the results are conveyed in a manner consistent with the SME practices and how will results be reported to support the project.

It was determined that a rank ordering of factor effects, with their relative contributions will be the first analysis performed. If the model is adequate, then subsequent analysis will identify factor combinations that provided the best (minimum) integrated drag.

11) Trial Runs: Can, or should, trial runs be conducted? When little prior experience is available with the experimental apparatus or system it is recommended to 
perform trial experiments to gain insight into a variety of the categories already discussed especially restrictions that can have a large impact on the design.

It was determined for this activity that based on the timeframe of running the experiment and previous experience with the CFD analysis code, that no trial runs would be executed.

\section{Experimental Design}

Utilizing the information from the meetings with the SMEs, an experimental design was developed. Two independent designs based on the flare factor were developed, one design without the flare and one with the flare to help understand its effects independently based on historical data. By removing the flare and its associated factors, the design space reduces from seven factors to four. In order to determine main effects and possible two and three factor interactions a full factorial design $2^{4}$ was chosen. Table 5 lists the configurations for each experimental unit and the respective settings as output from the software package used for the design and analysis of the data, Design Expert (registered trademark of State-ease Inc., no endorsement implied). In addition, two center runs were added (since the Tip Shape factor is categorical, a center run is needed for each setting) to investigate possible curvature in the model space.

\begin{tabular}{|r|c|r|r|r|r|}
\hline & & \multicolumn{1}{|c|}{ Factor 1 } & \multicolumn{1}{|c|}{ Factor 2 } & \multicolumn{1}{|c|}{ Factor 3 } & Factor 4 \\
\hline \multicolumn{1}{|c|}{ Std } & Point & A:TowerLen & B:TowerDia & C:TipFineRatio & D:TipShape \\
\hline Order & Type & \multicolumn{1}{|c|}{ inches } & \multicolumn{1}{|c|}{ inches } & l/d ratio & \\
\hline 1 & Fact & 326 & 26 & 0.5 & ellipse \\
\hline 2 & Fact & 490 & 26 & 0.5 & ellipse \\
\hline 3 & Fact & 326 & 46 & 0.5 & ellipse \\
\hline 4 & Fact & 490 & 46 & 0.5 & ellipse \\
\hline 5 & Fact & 326 & 26 & 2 & ellipse \\
\hline 6 & Fact & 490 & 26 & 2 & ellipse \\
\hline 7 & Fact & 326 & 46 & 2 & ellipse \\
\hline 8 & Fact & 490 & 46 & 2 & ellipse \\
\hline 9 & Fact & 326 & 26 & 0.5 & sphere/cone \\
\hline 10 & Fact & 490 & 26 & 0.5 & sphere/cone \\
\hline 11 & Fact & 326 & 46 & 0.5 & sphere/cone \\
\hline 12 & Fact & 490 & 46 & 0.5 & sphere/cone \\
\hline 13 & Fact & 326 & 26 & 2 & sphere/cone \\
\hline 14 & Fact & 490 & 26 & 2 & sphere/cone \\
\hline 15 & Fact & 326 & 46 & 2 & sphere/cone \\
\hline 16 & Fact & 490 & 46 & 2 & sphere/cone \\
\hline 17 & Center & 408 & 36 & 1.25 & ellipse \\
\hline 18 & Center & 408 & 36 & 1.25 & sphere/cone \\
\hline
\end{tabular}

Table 5. Four-Factor Experiment Design without Flare

Summary of 4-Factor Design Characteristics:

- Full Factorial, all possible combinations at two-levels

- Full Resolution, allows for the estimation of:

○ Main Effects, Two-, Three-, and Four-factor Interactions 
- Response model capable of estimating:

$$
y=\beta+\sum_{i=1}^{p} \beta_{i} x_{i}+\sum_{i=1}^{p-1} \sum_{j=i+1}^{p} \beta_{i j} x_{i} x_{j}+\sum_{i=1}^{p-2} \sum_{j=i+1}^{p-1} \sum_{k=j+1}^{p} \beta_{i j k} x_{i} x_{j} x_{k}+x_{1} x_{2} x_{3} x_{4}+\varepsilon
$$

where, $y$ is the response (integrated drag), the $\beta$ 's are the unknown model coefficients calculated from the experimental data, the x's are the variables (factors), and $\varepsilon$ is a bias error within the CFD code, and $p$ is the number of variables (in this case four).

- Curvature can be detected with center points

- Orthogonal in factorial portion (without center points)

$\circ$ Allows for unique estimation of model parameters

\section{- Total of $16+2=\mathbf{1 8}$ configurations}

The second design included the flare and its associated factors making it a seven factor design. Based on the discussions with the SME's it was determined that understanding interactions up to the three-factor level would be sufficient for this phase of the study. Therefore, a $2^{7-1}$ one-half fractional factorial design was chosen. Table 6 lists the configurations for each experiment unit/run. As in the four-factor deign two center runs were added to investigate curvature.

\begin{tabular}{|c|c|c|c|c|c|c|c|c|}
\hline & & Factor 1 & Factor 2 & Factor 3 & Factor 4 & Factor 5 & Factor 6 & Factor 7 \\
\hline Std & Point & A:TowerLen & B:TowerDia & C:TipFineRatio & D:FlareDiaRatio & $\mathrm{E}:$ FlareAngle & F:FlareLoc & G:TipShape \\
\hline Order & Type & inches & inches & I/d ratio & $\%$ of TowD & deg & $\mathrm{ht} /$ TowLen & \\
\hline 1 & Fact & 326 & 26 & 0.5 & 1.5 & 25 & 0.4 & sphere/cone \\
\hline 2 & Fact & 490 & 26 & 0.5 & 1.5 & 25 & 0.4 & ellipse \\
\hline 3 & Fact & 326 & 46 & 0.5 & 1.5 & 25 & 0.4 & ellipse \\
\hline 4 & Fact & 490 & 46 & 0.5 & 1.5 & 25 & 0.4 & sphere/cone \\
\hline 5 & Fact & 326 & 26 & 2 & 1.5 & 25 & 0.4 & ellipse \\
\hline 6 & Fact & 490 & 26 & 2 & 1.5 & 25 & 0.4 & sphere/cone \\
\hline 7 & Fact & 326 & 46 & 2 & 1.5 & 25 & 0.4 & sphere/cone \\
\hline 8 & Fact & 490 & 46 & 2 & 1.5 & 25 & 0.4 & ellipse \\
\hline 9 & Fact & 326 & 26 & 0.5 & 2.5 & 25 & 0.4 & ellipse \\
\hline 10 & Fact & 490 & 26 & 0.5 & 2.5 & 25 & 0.4 & sphere/cone \\
\hline 11 & Fact & 326 & 46 & 0.5 & 2.5 & 25 & 0.4 & sphere/cone \\
\hline 12 & Fact & 490 & 46 & 0.5 & 2.5 & 25 & 0.4 & ellipse \\
\hline 13 & Fact & 326 & 26 & 2 & 2.5 & 25 & 0.4 & sphere/cone \\
\hline 14 & Fact & 490 & 26 & 2 & 2.5 & 25 & 0.4 & ellipse \\
\hline 15 & Fact & 326 & 46 & 2 & 2.5 & 25 & 0.4 & ellipse \\
\hline 16 & Fact & 490 & 46 & 2 & 2.5 & 25 & 0.4 & sphere/cone \\
\hline 17 & Fact & 326 & 26 & 0.5 & 1.5 & 45 & 0.4 & ellipse \\
\hline 18 & Fact & 490 & 26 & 0.5 & 1.5 & 45 & 0.4 & sphere/cone \\
\hline 19 & Fact & 326 & 46 & 0.5 & 1.5 & 45 & 0.4 & sphere/cone \\
\hline 20 & Fact & 490 & 46 & 0.5 & 1.5 & 45 & 0.4 & ellipse \\
\hline 21 & Fact & 326 & 26 & 2 & 1.5 & 45 & 0.4 & sphere/cone \\
\hline 22 & Fact & 490 & 26 & 2 & 1.5 & 45 & 0.4 & ellipse \\
\hline 23 & Fact & 326 & 46 & 2 & 1.5 & 45 & 0.4 & ellipse \\
\hline 24 & Fact & 490 & 46 & 2 & 1.5 & 45 & 0.4 & sphere/cone \\
\hline
\end{tabular}




\begin{tabular}{|c|c|c|c|c|c|c|c|c|}
\hline 25 & Fact & 326 & 26 & 0.5 & 2.5 & 45 & 0.4 & sphere/cone \\
\hline 26 & Fact & 490 & 26 & 0.5 & 2.5 & 45 & 0.4 & ellipse \\
\hline 27 & Fact & 326 & 46 & 0.5 & 2.5 & 45 & 0.4 & ellipse \\
\hline 28 & Fact & 490 & 46 & 0.5 & 2.5 & 45 & 0.4 & sphere/cone \\
\hline 29 & Fact & 326 & 26 & 2 & 2.5 & 45 & 0.4 & ellipse \\
\hline 30 & Fact & 490 & 26 & 2 & 2.5 & 45 & 0.4 & sphere/cone \\
\hline 31 & Fact & 326 & 46 & 2 & 2.5 & 45 & 0.4 & sphere/cone \\
\hline 32 & Fact & 490 & 46 & 2 & 2.5 & 45 & 0.4 & ellipse \\
\hline 33 & Fact & 326 & 26 & 0.5 & 1.5 & 25 & 0.8 & ellipse \\
\hline 34 & Fact & 490 & 26 & 0.5 & 1.5 & 25 & 0.8 & sphere/cone \\
\hline 35 & Fact & 326 & 46 & 0.5 & 1.5 & 25 & 0.8 & sphere/cone \\
\hline 36 & Fact & 490 & 46 & 0.5 & 1.5 & 25 & 0.8 & ellipse \\
\hline 37 & Fact & 326 & 26 & 2 & 1.5 & 25 & 0.8 & sphere/cone \\
\hline 38 & Fact & 490 & 26 & 2 & 1.5 & 25 & 0.8 & ellipse \\
\hline 39 & Fact & 326 & 46 & 2 & 1.5 & 25 & 0.8 & ellipse \\
\hline 40 & Fact & 490 & 46 & 2 & 1.5 & 25 & 0.8 & sphere/cone \\
\hline 41 & Fact & 326 & 26 & 0.5 & 2.5 & 25 & 0.8 & sphere/cone \\
\hline 42 & Fact & 490 & 26 & 0.5 & 2.5 & 25 & 0.8 & ellipse \\
\hline 43 & Fact & 326 & 46 & 0.5 & 2.5 & 25 & 0.8 & ellipse \\
\hline 44 & Fact & 490 & 46 & 0.5 & 2.5 & 25 & 0.8 & sphere/cone \\
\hline 45 & Fact & 326 & 26 & 2 & 2.5 & 25 & 0.8 & ellipse \\
\hline 46 & Fact & 490 & 26 & 2 & 2.5 & 25 & 0.8 & sphere/cone \\
\hline 47 & Fact & 326 & 46 & 2 & 2.5 & 25 & 0.8 & sphere/cone \\
\hline 48 & Fact & 490 & 46 & 2 & 2.5 & 25 & 0.8 & ellipse \\
\hline 49 & Fact & 326 & 26 & 0.5 & 1.5 & 45 & 0.8 & sphere/cone \\
\hline 50 & Fact & 490 & 26 & 0.5 & 1.5 & 45 & 0.8 & ellipse \\
\hline 51 & Fact & 326 & 46 & 0.5 & 1.5 & 45 & 0.8 & ellipse \\
\hline 52 & Fact & 490 & 46 & 0.5 & 1.5 & 45 & 0.8 & sphere/cone \\
\hline 53 & Fact & 326 & 26 & 2 & 1.5 & 45 & 0.8 & ellipse \\
\hline 54 & Fact & 490 & 26 & 2 & 1.5 & 45 & 0.8 & sphere/cone \\
\hline 55 & Fact & 326 & 46 & 2 & 1.5 & 45 & 0.8 & sphere/cone \\
\hline 56 & Fact & 490 & 46 & 2 & 1.5 & 45 & 0.8 & ellipse \\
\hline 57 & Fact & 326 & 26 & 0.5 & 2.5 & 45 & 0.8 & ellipse \\
\hline 58 & Fact & 490 & 26 & 0.5 & 2.5 & 45 & 0.8 & sphere/cone \\
\hline 59 & Fact & 326 & 46 & 0.5 & 2.5 & 45 & 0.8 & sphere/cone \\
\hline 60 & Fact & 490 & 46 & 0.5 & 2.5 & 45 & 0.8 & ellipse \\
\hline 61 & Fact & 326 & 26 & 2 & 2.5 & 45 & 0.8 & sphere/cone \\
\hline 62 & Fact & 490 & 26 & 2 & 2.5 & 45 & 0.8 & ellipse \\
\hline 63 & Fact & 326 & 46 & 2 & 2.5 & 45 & 0.8 & ellipse \\
\hline 64 & Fact & 490 & 46 & 2 & 2.5 & 45 & 0.8 & sphere/cone \\
\hline 65 & Center & 408 & 36 & 1.25 & 2 & 35 & 0.6 & ellipse \\
\hline 66 & Center & 408 & 36 & 1.25 & 2 & 35 & 0.6 & sphere/cone \\
\hline
\end{tabular}

Table 6: Seven Factor Experiment Design with Flare

Summary of 7-Factor Design Characteristics:

- 1/2 Fraction of all possible factorial combinations

- Resolution VII, allows for estimation of:

○ Main Effects, Two- and Three-Factor Interactions 
- Response model capable of estimating

$$
y=\beta+\sum_{i=1}^{p} \beta_{i} x_{i}+\sum_{i=1}^{p-1} \sum_{j=i+1}^{p} \beta_{i j} x_{i} x_{j}+\sum_{i=1}^{p-2} \sum_{j=i+1}^{p-1} \sum_{k=j+1}^{p} \beta_{i j k} x_{i} x_{j} x_{k}+\varepsilon
$$

where, $y$ is the response (integrated drag), the $\beta$ 's are the unknown model coefficients calculated from the experimental data, the x's are the variables (factors), and $\varepsilon$ is a bias error within the CFD code, and $\mathrm{p}$ is the number of variables (in this case seven).

- Curvature detection capable, Orthogonal design

- Total of $64+2=\mathbf{6 6}$ configurations

\section{Experimental Results}

Each of the experimental runs was executed using the Overflow code (axisymmetric viscous). The results were entered into the integrated drag equation and a single coefficient was computed for each run. This data was then input into Design Expert software for analysis. This software package allows for graphical data exploration, model building and diagnostics. Figure 3 displays the results for the four-factor experiment. By partitioning the total variability in the response (integrated drag) into components that can be uniquely attributed to the factors and factor combinations, the large effects can be identified. As can be seen in the figure 3,93\% of the variability in the response (integrated drag) can be explained by four factors.

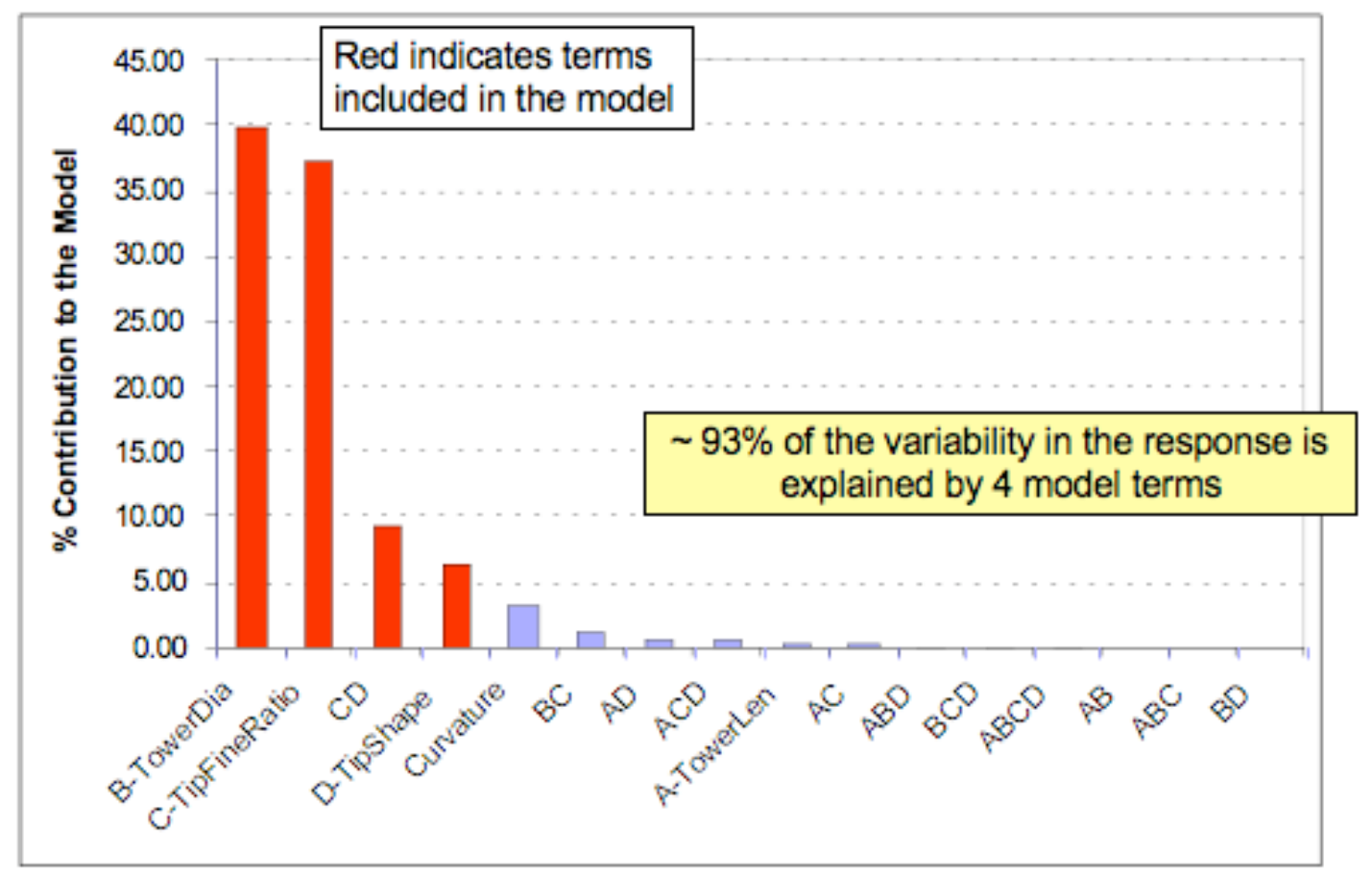

Figure 3. Four Factor Design Analysis Results - Large Effects Determined 
Figure 4 shows a plot of the interaction effect Tip Shape and Tip Fineness Ratio. The plot shows that Sphere/cone with a low fineness ratio minimizes integrated drag and the Sphere/cone is more sensitive to the tip fineness ratio than the elliptical shape. Curvature is detected since the line for the effect does not cross though or close-to the center data points represented by the red and black circles. The results of this analysis were successfully identifying the main and interaction effects as well as the settings for minimum drag, as listed below. Also, since curvature was detected, a higher order model could be generated if a predictive capability was desired. A summary of the results from the 4-factors experiment follow.

\section{Minimum Integrated Occurs when:}

B: Tower Dia. $=46$ (wide)

C: Tip Fineness Ratio $=0.5$ (blunt)

D: Tip Shape $=$ sphere/cone

A: Tower Len. - low contribution

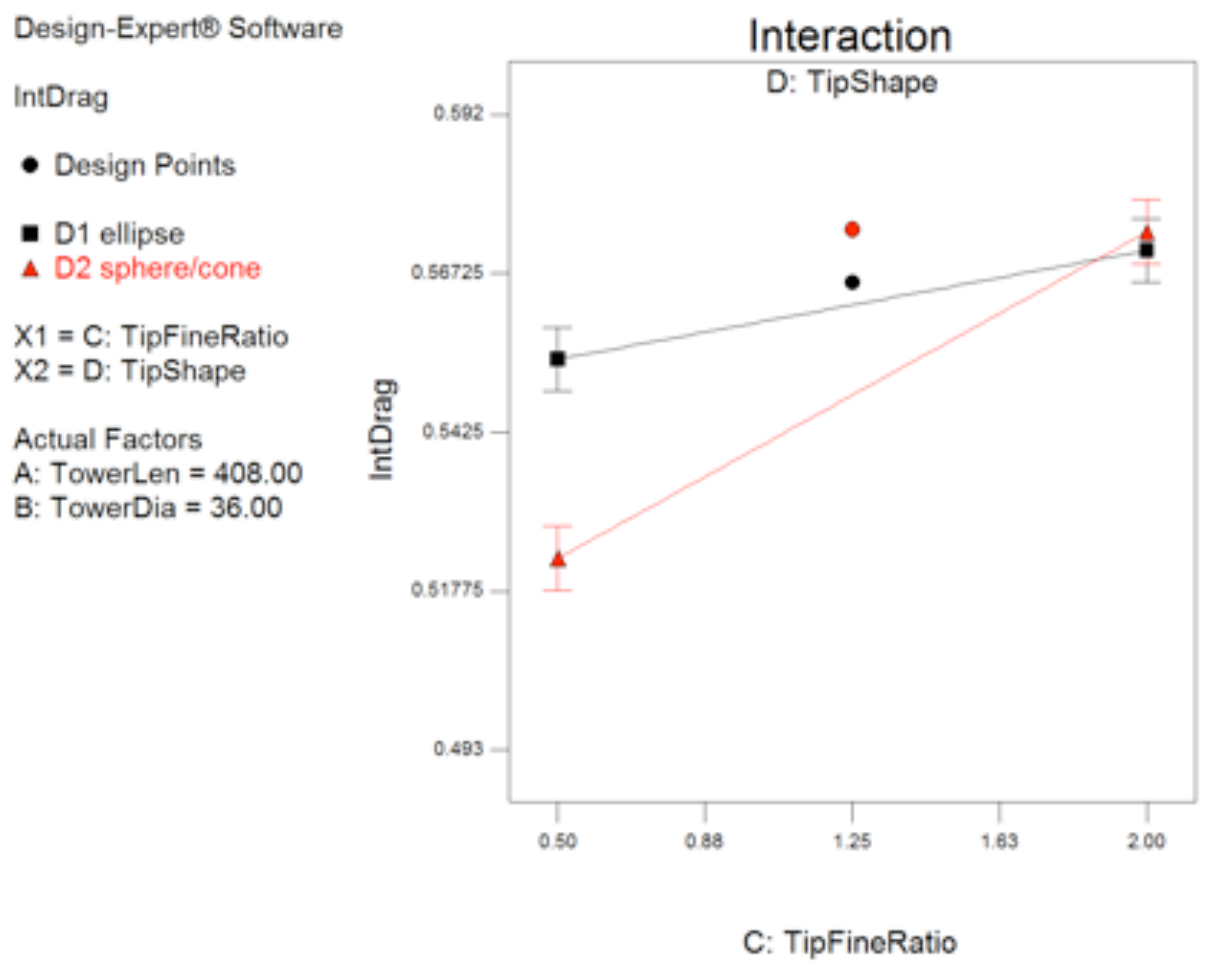

Figure 4. Interaction of Tip Shape with Tip Finess Ratio

The seven factor experiment analysis, where the flare is considered, followed the same analysis process and the resulting effects are shown in figure 5. Out of a possible 64 terms, five were able to explain $90 \%$ of the variability in the response. The combinations of the large effects for minimum drag are listed below. 


\section{Minimum Integrated Drag Occurs when:}
B: Tower Dia. $=46$ (wide)
F: Flare Location $=0.4$ (high)
D: Flare Dia. Ratio $=2.5$ (wide)
E: Flare Angle $=45$ (greater)
C: Tip Fineness Ratio $=0.5$ (blunt)

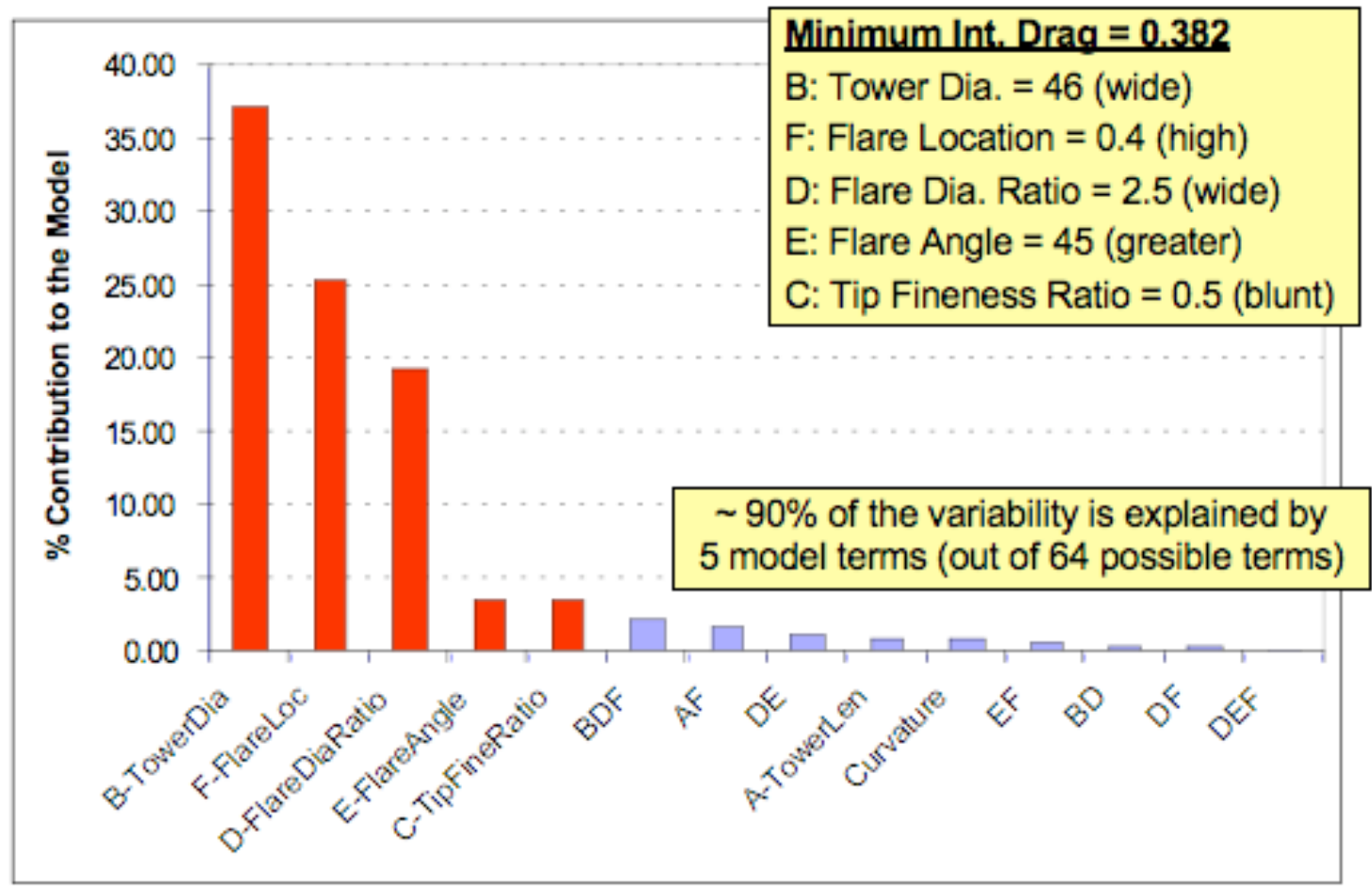

Figure 5. Seven Factor Design Analysis Results - Large Effects Determined

\section{Summary}

Design of Experiments (DOE) methodologies were applied to the LAS parametric geometry CFD study to efficiently identify and rank primary contributors to integrated drag over the vehicles ascent trajectory in an order of magnitude fewer CFD configurations thereby reducing computational resources and solution time. As a tool being utilized by the Ares-I CLV Aerodynamics Panel to perform rapid assessments of possible OML design changes, CFD combined with DOE enabled efficient down-select to the important parameters. In addition, SME's were able to gain a better understanding on the underlying flow-physics of different geometric parameter configurations through the identification of interaction effects. An interaction effect, which describes how the effect of one factor changes with respect to the levels of other factors, is often the key to product optimization. A DOE approach emphasizes a sequential approach to learning through successive experimentation to continuously build on previous knowledge. These studies represent a starting point for expanded experimental activities that will eventually cover the entire design space of the vehicle and flight trajectory. 


\section{Acknowledgements}

The authors would like to thank Bob Hall (NASA Langley Research Center) of the CLV Aerodynamics Team for providing the opportunity to apply DOE to this activity, and Pieter Buning and his CFD Team (NASA Langley Research Center) for performing the CFD analysis.

\section{References}

1. Huebner, L.D., et.al.; "Status, Plans, and Initial Results for Ares I Crew Launch Vehicle Aerodynamics," IAC-06-D2.7.03; 57 ${ }^{\text {th }}$ International Astronautical Congress, Valencia, Spain; October, 2006.

2. Montgomery, Douglas C; Design and Analysis of Experiments $5^{\text {th }}$ Edition; 2002.

3. Coleman, D.E. and Montgomery, D.C. (1993), "A Systematic Approach to Planning for a Designed Industrial Experiment," (with Discussion), Technometrics, Vol. 35, pp. 1-27. 